Pacific Journal of Mathematics

MACKEY ANALYSIS OF INFINITE CLASSICAL MOTION 


\title{
MACKEY ANALYSIS OF INFINITE CLASSICAL MOTION GROUPS
}

\section{Doug Pickrell}

\begin{abstract}
Representation theory for infinite classical motion groups is formulated in terms of invariant measure classes and cocycle cohomology. It is shown that invariant measure classes are always represented by invariant probability measures, and these classes are determined for Cartan motion groups. The existence of "induced" cocycle cohomology is established in this ergodic setting. Also it is shown that the continuity properties of representations are rather rigidly determined.
\end{abstract}

1. Introduction. Let $K \widetilde{\times} A$ denote a semidirect product of separable topological groups, where $A$ is abelian. If both $K$ and $A$ are locally compact, then it can be shown that every separable unitary representation of $K \widetilde{\times} A$ can be uniquely written as a direct integral of factor representations of the following form: the Hilbert space is of the form

$$
H=L^{2}\left(A^{\wedge}, \mu\right) \otimes \mathscr{H}_{m},
$$

where $A^{\wedge}$ is the character group of $A, \mu$ is an ergodic $K$-quasiinvariant Borel measure, and $\mathscr{H}_{m}$ is an $m$-dimensional Hilbert space $(1 \leq m \leq \infty)$; the action is given by

$$
((x, g) \cdot F)(\chi)=\chi(x) c(g, \chi)\left[\frac{d \mu\left(g^{-1} \chi\right)}{d \mu(\chi)}\right]^{1 / 2} F\left(g^{-1} \chi\right) \text {, }
$$

for $x \in A, g \in K$, where $c$ is a unitary cocyle. Mackey showed that if $\mu$ is concentrated on a $K$-orbit, then the above representation is equivalent to an induced representation-a tremendous simplication. This leads to a relatively complete understanding of the representation theory for $K \widetilde{\times} A$, at least in situations where all the $K$-ergodic measures on $A^{\wedge}$ are transitive.

The goal of this paper is to carry through some steps of the above analysis for a class of infinite dimensional motion groups: representations are canonically decomposed into factors, the factors are realized as $L^{2}$ spaces, $K$-invariant measure classes on $A^{\wedge}$ are determined, and large classes of cocycles are described.

The principal difference between the finite and infinite dimensional theories is that for the latter $K$-ergodic measures are essentially always purely ergodic. Despite this (and finite dimensional intuition), 
there are good reasons to believe that a cohomological classification of cocycles is feasible (in particular Ol'shanskii has shown that finite rank Cartan motion groups are type I). But a complete result has not been attained for a single example.

The groups considered in this paper (modulo a technical reformulation discussed below) are of the form $K \widetilde{\times} H$, where $K$ is a finite product of rotation groups of separable $(\mathbb{R}, \mathbb{C}$ or $\mathbb{H})$ Hilbert spaces, and $H$ is a separable representation of $K$. The groups $K$ can be characterized in the following way: they are the automorphism groups of finite rank "compact type" Riemannian symmetric spaces. Because of this finiteness, these groups are similar, in some respects, to compact groups (in particular they have discrete spectra).

Section 2 of this paper contains a brief review of the representation theory of groups $K$. The problem is also slightly reformulated: we consider representations for an inductive limit subgroup $K(\infty) \widetilde{\times} H(\infty)$ of $K \widetilde{\times} H$. This is technically convenient, but also, as it turns out, for certain semidirect products we can prove that representations for $K(\infty) \tilde{\times} H(\infty)$ automatically extend to $K \widetilde{\times} H$ (see (6.1) for a precise statement). This has important consequences for anomalous commutation relations in quantum field theory (see $\S 6$ and [Pi5]).

Aside from $\S 6$, the outline of the paper is straightforward. In $\S 3$ representations for $K(\infty) \tilde{\times} H(\infty)$ are realized in terms of measures and cocycles on $H(\infty)^{\wedge}$, as in finite dimensions. A key fact about the groups $K$ is that their invariant measure classes are always represented by invariant probability measures. This is presented in $\S 4$.

In $\S 5$ we consider $K$-invariant measures for linear actions. In finite dimensions every ergodic measure for a compact group is an equivariant image of Haar measure. This is very useful for cocycle analysis, for any cocycle can then be pulled back to the standard affine action of the group on itself (this is true more generally for any transitive action of a separable locally compact group, and this technique is central to Mackey analysis). For $K$ there is a natural analogue of Haar measure-a Gaussian measure on an appropriate space. It is not true that every linear ergodic action is an image of this measure. However it appears to be true in an approximate sense for linear actions (which is made precise). The point of this section is that we can actually classify the ergodic probabilities in the most important cases. The general picture we envision is true in these cases. In particular this is carried out for Cartan motion actions. In the infinite rank case it turns out that this classification result is essentially equivalent to Schoenberg's classification of Polya frequency functions, together 
with a result of Karlin in the general theory of total positivity. These classification results are critical to understanding harmonic analysis on infinite dimensional symmetric spaces, which is pursued in [Pi6]. The implications for random matrix theory are unclear at this point.

In the last section we present examples of cocycles. It appears that finite rank cocycles behave as in finite dimensions. Our expectation is that the finite rank cocycle cohomology for linear actions on Gaussians (our substitute for Haar measure) will be zero, while for nonlinear images of Gaussians the finite rank cohomology will be fully accounted for by an induction process.

Infinite rank cocycle cohomology appears to behave in an opposite manner. It appears that the infinite rank cohomology is relatively complicated for linear actions on Gaussians, because cocycles pulled back from equivariant images do not simplify. Our expectation is that as one passes down the tree of equivariant maps, the infinite rank cohomology simplifies. This is all pure speculation however-we do not have any complete classification results.

NotAtion. In this paper, in dealing with representations of a semidirect product $K \widetilde{\times} A$, it will be convenient to let $K$ act first. Thus we will view $K \widetilde{\times} A$ as the set $A \times K$ with the multiplication

$$
(x, g)(y, h)=(x(g \cdot y), g h) \text {. }
$$

2. Infinite rotation groups. In this section we will briefly recall the separable unitary representation theory of the class of groups which are finite products of rotation groups of separable real, complex, or quaternionic Hilbert space. This theory is mainly due to Kirillov and Ol'shanskii ([Kir], [Ol1]). At the end of the section we will then be in a position to say precisely what representations we intend to study in the remainder of the paper.

This class of groups can be characterized in at least two ways. On the one hand it can be viewed as the class of automorphism groups of the finite rank "compact type" Riemannian symmetric spaces without compact factors (the irreducible spaces of this type are precisely the Grassmannians $\operatorname{Gr}\left(n, H_{\mathbb{R}}\right), \operatorname{Gr}\left(n, H_{\mathbb{C}}\right), \operatorname{Gr}\left(n, H_{\mathbb{H}}\right)$ and the space of real oriented $n$-planes $(n<\infty))$. On the other hand it is identical to the class of groups which arise as isotropy groups for finite products of irreducible infinite rank Riemannian symmetric spaces. From both points of view one would expect these groups to be much like compact groups. 
We will use the symbol $K(\infty)$ to denote the finite product of the groups $\mathrm{SO}(\infty, \mathbb{R}), \mathrm{U}(\infty, \mathbb{C})$ and $\mathrm{Sp}(\infty)$. If $K(\infty)=\mathrm{SO}(\infty, \mathbb{R})$, then we will assume there is a fixed real Hilbert space $H$ and a distinguished orthonormal basis $\varepsilon_{1}, \varepsilon_{2}, \ldots$, so that $K(\infty)=\bigcup_{n} \mathrm{SO}(n, \mathbb{R})$, where we identify $K(n)=\mathrm{SO}(n, \mathbb{R})$ with those special orthogonal transformations that fix $\varepsilon_{n+1}, \varepsilon_{n+2}, \ldots$ In this case $K$ will equal $\mathrm{O}(H)$, and $K_{\infty}=\{g \in \mathrm{O}(H): g=1+$ compact operator $\}$. Thus in this case, the closure of $K(\infty)$ in the uniform operator topology will equal $\left(K_{\infty}\right)_{0}$, the identity component of $K_{\infty}$ in the uniform topology. If $K(\infty)=\mathrm{U}(\infty, \mathbb{C}), K$ will equal $\mathrm{U}(H)$, where $H$ is now a complex Hilbert space with a distinguished orthonormal basis, and so on (if $K(\infty)$ is a product, $K(n), K$ and $K_{\infty}$ will denote the corresponding products viewed as groups of operators on the corresponding product of Hilbert spaces).

The class of representations of $K(\infty)$ which we will consider is very special, as our first proposition will show. We have natural maps of topological groups

$$
\left(\left(K_{\infty}\right)_{0}, \tau_{u}\right) \rightarrow\left(K, \tau_{u}\right) \rightarrow\left(K, \tau_{s}\right) \cong\left(K, \tau_{w}\right)
$$

and

$$
\left(K, \tau_{d}\right) \rightarrow\left(K, \tau_{n}\right)
$$

where $\tau_{u}=$ uniform topology, $\tau_{s}=$ strong topology, $\tau_{w}=$ weak topology, and $\tau_{d}=$ discrete topology.

(2.1) Proposition. The natural maps $\left(K, \tau_{w}\right)^{\wedge} \cong\left(K, \tau_{s}\right)^{\wedge} \rightarrow$ $\left(K, \tau_{u}\right)^{\wedge} \rightarrow\left(\left(K_{\infty}\right)_{0}, \tau_{u}\right)^{\wedge}$ are bijections, where $G^{\wedge}$ denotes the set of equivalence classes of strongly continuous separable unitary representations of the topological group $G$.

We also conjecture that both

$$
\left(K, \tau_{s}\right)^{\wedge} \rightarrow\left(K, \tau_{d}\right)^{\wedge} \text { and }\left(K_{\infty}, \tau_{u}\right)^{\wedge} \rightarrow\left(K_{\infty}, \tau_{d}\right)^{\wedge}
$$

are bijective.

(2.2) Definition. A strongly, continuous separable unitary representation for $K(\infty)$ (or $K$ or $K_{\infty}$ ) equipped with the strong uniform topology will be called tame. A $K(\infty)$-quasi-invariant measure will be called tame if the corresponding $L^{2}$ representation is tame.

A fairly direct proof of (2.1) can be given in the following way. To see that the first arrow is bijective, we observe that the strong and 
uniform topologies induce the same Borel structure on $K$. This is so because

$$
\left\{g \in K:|g-1|_{\infty}<\varepsilon\right\}=\bigcap_{j}\left\{g \in K:\left|g \cdot x_{j}-x_{j}\right|<\varepsilon\left|x_{j}\right|\right\}
$$

where $\left\{x_{j}\right\}$ is the countable dense set in our underlying product of $(\mathbb{R}, \mathbb{C}$ and $\mathbb{H})$ Hilbert spaces. Now suppose that $\pi \in\left(K, \tau_{u}\right)^{\wedge}$. Then $\pi$ will be a Borel homomorphism from $\left(K, \tau_{s}\right)$ to $\left(\mathrm{U}(H(\pi)), \tau_{s}\right)$. Since these are Polish topological groups, $\pi$ is actually continuous, by a theorem of Banach ([Mo]). This proves the first arrow is bijective.

Since $K(\infty)$ is dense in $\left(K, \tau_{s}\right)$, this argument also shows the second arrow is injective.

The proof that the second arrow is surjective depends upon a lemma of Kirillov, which actually gives another useful characterization of tame representations. If $K(\infty)$ consists of a single factor, we let $K(\infty)_{n}$ denote the subgroup that fixes each of the first $n$ basis elements; in general it denotes the corresponding product.

(2.3) LEMMA. If $\pi \in\left(K_{\infty}\right)^{\wedge}$, then $H(\pi)^{K(\infty)_{n}} \neq\{0\}$ for some $n$.

A short proof can be found in [011].

To complete the proof of $(2.1)$, suppose $\pi \in\left(K_{\infty}\right)^{\wedge}$. For definiteness suppose $K=\mathrm{U}(H)$. By (2.3) we can assume there is a cyclic vector $v \in H(\pi)^{K(\infty)_{n}}$. The positive definite function $\langle\pi(g) v, v\rangle$ depends only upon $g \varepsilon_{1}, \ldots, g \varepsilon_{n}$; hence $\langle\pi(g) v, v\rangle$ is continuous for the strong topology. Since $\mathrm{U}(\infty, \mathbb{C})$ is dense in $\left(\mathrm{U}(H), \tau_{s}\right)$, this implies $\pi \in\left(K, \tau_{s}\right)^{\wedge}$.

To describe the tame representations, we first consider the special case of $K=\mathrm{U}(H)$, which is of special interest.

(2.4) Definition. A tame representation $T$ of $K$ is holomorphic if it extends to a holomorphic representation of $\mathscr{C}=(\{L \in \mathscr{L}(H)$, $\left.\left.|L|_{\infty}<1\right\}, \tau_{w}\right)$ i.e., $\mathscr{C} \rightarrow \mathscr{C}(H(T))$ is a morphism and matrix coefficients are holomorphic.

I. Segal ([Se]) proved that (just as in finite dimensions) the holomorphic representations decompose discretely and the irreducible holomorphic representations are precisely those obtained by decomposing the tensor algebra $\mathscr{T}(H)$. It follows coherently from finite dimensional considerations that $\mathrm{U}(H)$ and $\operatorname{Perm}(n)$ act as a dual pair on $\mathscr{T}^{n}(H)$, so that the irreducible holomorphic representations are naturally parameterized by partitions (this is also consistent with the orbit method and Borel-Weil ([Bo])). 
To round out the picture, consider the embedding $K \rightarrow \widetilde{K}$, where $\mathrm{U}(H) \rightarrow \mathrm{U}(H) \times \mathrm{U}(H): g \rightarrow(g, \bar{g}), \quad \mathrm{O}(H) \rightarrow \mathrm{U}\left(H_{\mathbb{C}}\right), \quad \mathrm{Sp}\left(H_{\mathbb{H}}\right) \rightarrow$ $\mathrm{U}\left(H_{\mathbb{C}}\right)$.

(2.5) Proposition. Every tame representation $T$ of $K$ extends uniquely to a holomorphic representation $\widetilde{T}$ of $\widetilde{K}$. Here $H(T)=H(\widetilde{T})$ and $T^{\prime}=\widetilde{T}^{\prime}$ (equality of commutants).

This point of view is advocated by Ol'shanskii in [O12] (from which the ideas below are drawn). Note that for $K=\mathrm{U}(H)$, this shows that the separable representations of $K$ are in bijective correspondence with holomorphic contraction representations of $\mathscr{C} \times \mathscr{C}$. This should be compared with "Wick rotation" in conformal field theory, where a similar correspondence is conjectured to exist with $\operatorname{Diff}^{+}\left(S^{1}\right)$ in place of $\mathrm{U}(H)$ and a certain semigroup of Riemann surfaces in place of $\mathscr{C}$ (see [Se2]).

If $K$ does not contain unitary factors, then the proof is quite short. Suppose $K=\mathrm{O}(H)$ for definiteness. By finite dimensional theory there is a holomorphic extension of $T: \mathrm{SO}(\infty, \mathbb{R}) \rightarrow \mathrm{U}(H(T))$ to a morphism

$$
T^{\mathbb{C}}:\left\{g \in \mathrm{SO}(\infty, \mathbb{C}):|g|_{\infty} \leq 1\right\} \rightarrow\left\{A \in \mathscr{L}(H(T)):|A|_{\infty} \leq 1\right\} .
$$

Since domain $\left(T^{\mathbb{C}}\right)$ is weakly dense in $\left\{L \in \mathscr{L}\left(H^{\mathbb{C}}\right):|L|_{\infty} \leq 1\right\}$, to prove existence and uniqueness of $\widetilde{T}$, it suffices to prove $T^{\mathbb{C}}$ is weakly continuous for $\left(\operatorname{domain}\left(T^{\mathbb{C}}\right), \tau_{w}\right)$. This follows from Kirillov's lemma (2.3), since it guarantees existence of a dense set of $\xi \in$ $H(T)$ such that the matrix coefficient $\langle T(g) \xi, \xi\rangle$ depends only upon a finite number of matrix coefficients of $g \in \mathrm{SO}(\infty, \mathbb{R})$. By holomorphy the same thing is true for $\left\langle T^{\mathbb{C}}(g) \xi, \xi\right\rangle$ for $g \in \mathrm{SO}(\infty, \mathbb{C})$.

To prove equality of commutants, suppose $V \subseteq H(T)$ is $T$ invariant. $V$ is then invariant for the extension of $T$ to $\{L \in \mathscr{L}(H):|L| \leq$ 1\}. Complex analyticity of matrix coefficients then implies that $V$ is invariant under $\left\{L \in L\left(H^{\mathbb{C}}\right):|L|_{\infty} \leq 1\right\}$ (for if $\xi \in V, \eta \in V^{\perp}$, $\langle L \cdot \xi, \eta\rangle$ vanishes on real operators implies it vanishes on complex operators). This implies $V$ is $\widetilde{T}$ invariant. Thus $T^{\prime}=\widetilde{T}^{\prime}$.

This proof also clearly works for $\operatorname{Sp}(H)$. The argument is slightly more complicated if $K=\mathrm{U}(H)$ (see [Ol1]).

It follows from (2.5) that tame irreducible representations for $K(\infty)$ are parameterized by sequences of partitions, one for each orthogonal and symplectic factor, two for each unitary factor. We will refer to 
the sum of the lengths of these partitions as the degree of the representation. An irreducible holomorphic representation for $\mathrm{U}(H)$ has degree $d$ if and only if it is a summand of $\mathscr{T}^{d}(H)$. Also we say a representation is homogeneous of degree $d$ if each irreducible summand has degree $d$. Proposition (2.5) has the following

(2.6) CoRollaRy. If $T_{j}$ is homogeneous of degree $d_{j}$, then $T_{1} \otimes T_{2}$ is homogeneous of degree $d_{1}+d_{2}$.

If $\pi$ is a real orthogonal representation of $K(\infty)$, we will say $\pi$ is tame if the action on $H(\pi)^{\mathbb{C}}$ is tame. Such representations are discretely decomposable.

The basic setting of the remainder of the paper is now described by the following

(2.7) Definition. If $\pi$ is a tame real orthogonal representation of $K(\infty)$ with finite multiplicities, then we set

$$
H(\pi)_{0}=\bigcup_{n} H(\pi)^{K(\infty)_{n}}
$$

with the inductive limit topology. We then say that a separable unitary representation of $K(\infty) \tilde{\times} H(\pi)_{0}$ is admissible if its restriction to $K(\infty)$ is tame.

The ultimate goal of this work is to determine all admissible representations. We are not working directly with representations of $K \widetilde{\times} H(\pi)$, because we want to prove that under appropriate conditions admissible representations automatically extend to $K \widetilde{\times} H(\pi)$ (see $\S 6$ ).

Appendix. Some other products. The products we are considering are analogous to products of the form $\mathscr{K} \widetilde{\times} \mathbb{R}^{n}$, where $\mathscr{K}$ is compact. There is also an analogue for real semisimple Lie groups, to which must of this paper may apply. We will occasionally refer to this in the sequel.

Suppose $G$ is the identity component of the automorphism group of an irreducible infinite rank Riemannian symmetric space, $K$ a stability subgroup. Then $(G, K)$ contains a covering of an inductive limit pair $(G(\infty), K(\infty))$, where each $(G(n), K(n))$ is a classical symmetric pair of rank $n$ with the usual embeddings (these are listed in (5.11) below-the point here is that there are no exceptional infinite dimensional symmetric spaces). For simplicity suppose $G(\infty) \subseteq G$.

Now suppose $\pi$ is a separable real orthogonal representation of $G$ with finite $K$-multiplicities. Representations of $G$ are determined by 
their restrictions to $G(\infty)$ (see $\S 5$ of [P4]). The analogue of (2.7) for $G \tilde{\times} H(\pi)$ is $G(\infty) \tilde{\times} H(\pi)_{0}$, where $H(\pi)_{0}=\bigcup_{n} H(\pi)^{K(\infty)_{n}}$. Here a separable unitary representation is admissible for $G(\infty) \widetilde{\times} H(\pi)_{0}$ if its restriction to $K(\infty) \subseteq G(\infty)$ is tame.

Examples of how these semidirect products arise in field theory can be found in [MR] and [Mi].

Other examples of semidirect products that arise in current algebra include $\mathscr{G} \tilde{\times} \operatorname{Affine}(\mathscr{A} ; \mathbb{R})$ and $\operatorname{Diff}(X) \tilde{\times} C^{\infty}(X ; \mathbb{R})$. Here $X$ is a compact manifold, $\mathscr{G}=\operatorname{map}(X, G)$ is a gauge group, and $\mathscr{A}$ is a space of $G$-connections (again see [Mi]). The sort of questions we are asking in this paper arise naturally in the context of field theory for these groups, but essentially nothing is known about how to attack them.

3. Preliminary Mackey analysis. Let $\rho$ be an admissible representation of $K(\infty) \tilde{\times} H(\pi)_{0}$ as in (2.7). A slight adaptation of an argument of Ol'shanskii's (Theorem 3.6 of [Ol1]) leads to the following

(3.1) Proposition. $\rho$ has a unique decomposition as a direct integral of admissible factor representations for $K(\infty) \widetilde{\times} H(\pi)_{0}$.

Proof. Let $\rho^{\prime}$ denote the commutant. Decompose $H(\rho)$ relative to the abelian algebra $\rho^{\prime} \cap \rho^{\prime \prime}: H(\rho)=\int \oplus H(\zeta) d \nu(\zeta)$. Because $K(\infty) \tilde{\times}$ $H(\pi)_{0}$ is a countable union of locally compact groups, if $\rho(g)=$ $\int \rho_{\zeta}(g) d \nu(\zeta)$ for $g \in K(\infty) \tilde{\times} H(\pi)_{0}$, then for a.e. $\zeta, \rho_{\zeta}$ is a unitary representation for $K(\infty) \tilde{\times} H(\pi)_{0}$, and $\rho=\int \rho_{\zeta} d \nu(\zeta)$ as representations. Thus the existence and uniqueness of the decomposition into factors follows from finite dimensional considerations.

The novel point is that the factors of the decomposition are admissible (almost everywhere). To see this let $P_{n}$ denote the orthogonal projection onto $H(\rho)^{K(\infty)}$ (as in (2.3)). We have $P_{n} \in \rho^{\prime \prime}$. For if $\lambda_{k}$ is the normalized Haar measure for $K(k)$, then $\rho\left(\lambda_{k}\right) \downarrow P_{0}$ strongly. Thus $P_{0} \in \rho^{\prime \prime}$. A similar argument implies $P_{n} \in \rho^{\prime \prime}$. Now write $P_{n}=\int P_{n}(\zeta) d \nu(\zeta)$. Then clearly $P_{n}(\zeta) H(\zeta)=H(\zeta)^{K(\infty)_{n}}$ for a.e. $\zeta$, and $P_{n} \uparrow 1$ strongly in $H(\rho)$ if and only if $P_{n}(\zeta) \uparrow 1$ strongly in $H(\zeta)$ for a.e. $\zeta$. Thus $H(\rho)$ is admissible if and only if $H(\zeta)$ is admissible for a.e. $\zeta$.

(3.2) REMARK. (3.1) is also valid for the more general class of semidirect products $G(\infty) \widetilde{\times} H(\pi)_{0}$ described in the Appendix of $\S 2$. An interesting question is whether (3.1) is valid for $\mathscr{G} \widetilde{\times} \operatorname{Affine}(\mathscr{A}, \mathbb{R})$ or 
$\operatorname{Diff}(X) \widetilde{\times} C^{\infty}(X ; \mathbb{R})$. The remainder of this section does apply to these groups.

In the remainder of this section we need to analyze an admissible factor representation $\rho$ for $K(\infty) \tilde{x} H(\pi)_{0}$. The upshot is (3.7) below, which is a consequence of the very general considerations to follow.

Suppose $A$ is a separable topological vector group satisfying the following

(3.3) Hypothesis. Every continuous positive definite function on $A$ is the Fourier transform of a unique measure on $A^{\wedge}$.

This is satisfied for instance if $A=H(\pi)_{0}$ (with the inductive limit topology), by the Kolmogorov extension theorem, or if $A$ is a nuclear space, by the theorem of Minlos. It is not satisfied if $A$ is a Hilbert space.

This hypothesis is really equivalent to saying that $A$ has a "good" representation theory.

(3.4) Proposition. If $A$ satisfies (3.3), then the separable strongly continuous unitary representations of $A$, up to equivalence, are in bijective correspondence with sequences of mutually disjoint measure classes on $A^{\wedge}$, where if $\left\{\left[\nu_{n}\right]\right\}$ is such a sequence

$$
H(\pi)=\sum \oplus L^{2}\left(A^{\wedge}, \nu_{n}\right) \otimes \mathscr{H}_{n},
$$

$\operatorname{dimn}\left(\mathscr{H}_{n}\right)=n$, and $A$ acts by multiplication:

$$
\left.\pi_{n}(x)(\Phi)\right|_{\zeta}=e^{i\langle x, \zeta\rangle} \Phi(\zeta)
$$

For $A$ locally compact this is the usual spectral theorem. The point here is that (3.3) guarantees that the same argument applies in this more general setting.

Now suppose that $\mathscr{K}$ is a topological automorphism group of $A$. If $\rho$ is a representation of $\mathscr{K} \widetilde{\times} A, \pi=\rho \mid A$, then $\mathscr{K}$ fixes the measure class of each $\nu_{n}$ in (3.4), and in $H\left(\pi_{m}\right)=L^{2}\left(A^{\wedge}, \nu_{m}\right) \otimes \mathscr{H}_{m}$,

$$
\left.\rho(x, k)(\Phi)\right|_{\zeta}=e^{i\langle x, \zeta\rangle} c_{m}(k, \zeta) \Phi\left(k^{-1} \cdot \zeta\right) \text { a.e. } \zeta,
$$

where $c_{m}(k, \cdot): A^{\wedge} \rightarrow U\left(\mathscr{H}_{m}\right)$ is $\nu_{m}$-measurable and $c_{m}\left(k_{1}, k_{2}\right)=$ $c_{m}\left(k_{1}\right)\left(k_{1} \cdot c_{m}\left(k_{2}\right)\right)$.

To make a precise statement about the classification of cocycles, we recall some results from [Mo]. Equip $\mathrm{U}\left(\mathscr{H}_{m}\right)$ with the strong topology. If $X$ is a countably generated Borel space and $\nu$ a finite Borel measure 
on $X$, we let $F=F_{\nu}\left(X, \mathrm{U}\left(\mathscr{H}_{m}\right)\right)$ denote the space of equivalence classes of $\nu$-measurable functions, equivalence meaning equality a.e. $\nu$. The topology of $F$ is defined by the metric

$$
\bar{\omega}(f, g)=\int \omega(f(x), g(x)) d_{\widetilde{F}}(x),
$$

where $\omega$ is a metric for $\left(\mathrm{U}\left(\mathscr{H}_{m}\right), \tau_{s}\right)$ with finite diameter. $F$ is then a Polish group (for pointwise multiplication), and the topology depends only on the measure class of $\nu$ and not on $\omega$.

Also, if $\mathscr{K}$ is a topological automorphism group of $F$, then $H^{1}(\mathscr{K}, F)$ is the set of continuous crossed homomorphisms $(c(g h)=$ $c(g)(g \cdot c(h)))$ modulo the equivalence relation $c_{1} \sim c_{2} \leftrightarrow$ there is $f \in F$ such that $f c_{1}(k)=c_{2}(k)(k \cdot f)$ for all $k \in \mathscr{K}$.

(3.6) Proposition. Let $\mathscr{K}$ be a topological automorphism group of $A, A$ satisfying the hypothesis (3.3). The separable strongly continuous unitary representations $\pi$ of $\mathscr{K} \widetilde{\times} A$, up to equivalence, are in bijective correspondence with sequences $\left\{\left(\left[\nu_{m}\right],\left[c_{m}\right]\right)\right\}$, where the $\left[\nu_{m}\right]$ are mutually disjoint $\mathscr{K}$-invariant measure classes on $A^{\wedge}$, and $\left[c_{m}\right] \in H^{1}\left(\mathscr{K}, F_{\nu_{m}}\left(A^{\wedge}, \mathrm{U}\left(\mathscr{H}_{m}\right)\right)\right)$. (The representation corresponding to this data is given by (3.5).)

Note that if the representation $\pi$ is a factor, then $\pi=\pi_{m}$ and $\nu_{m}$ is ergodic.

(3.7) COROllaRY. If $\rho$ is an admissible factor representation of $K(\infty) \widetilde{\times} H(\pi)_{0}$, then there is a uniquely determined $m$, a tame ergodic $K(\infty)$-invariant measure class $[\nu]$ on $H(\pi)_{0}^{\wedge}$, and a cocycle $[c] \in$ $H^{1}\left(K, F_{\nu}\left(H(\pi)_{0}^{\wedge}, \mathrm{U}\left(\mathscr{H}_{m}\right)\right)\right)$ such that $H(\rho)=L^{2}\left(H(\pi)_{0}^{\wedge}, \nu\right) \otimes \mathscr{H}_{m}$ and $\rho$ acts by (3.5).

4. Quasi-invariant measures. Recall we say that a quasi-invariant measure for $K(\infty)$ is tame if the corresponding natural $L^{2}$ representation is tame (see (2.2)).

(4.1) Proposition. Suppose $K(\infty)$ acts on a standard Borel space $X$. If $\mu$ is a tame quasi-invariant measure for this action, then $\mu$ is equivalent to an invariant probability measure.

This result depends strongly on the assumption of tameness. We will discuss some examples after giving the proof. 
Proof of (4.1). We can assume that $\mu$ is a probability measure. For each $g \in K(\infty)$ there is a Borel function $\alpha(g, \cdot)$ on $X$ such that

$$
\alpha(g, x) d \mu(x)=d \mu\left(g^{-1} x\right) .
$$

Our assumption that $\mu$ is tame means that the natural representation on $L^{2}(X, \mu)$,

$$
\pi(g) \psi=\alpha(g, x)^{1 / 2} \psi\left(g^{-1} x\right)
$$

is continuous for $K(\infty)$ in the strong operator topology.

To prove (4.1) it suffices to show that $L^{2}(X, \mu)^{K(\infty)}$ is nontrivial. For if $\psi$ is invariant and nontrivial then $|\psi|$ and $\{x:|\psi|>0\}$ are invariant. On this set of positive $\mu$-measure, the restriction $\mu_{+}$is equivalent to the invariant finite measure $|\psi|^{2} \mu_{+}$. We now consider the complement and continue reducing the mass, if any remains.

For each $n$ let $Q_{n}$ denote the orthogonal projection onto $L^{2}(X, \mu)^{K(n)}$. We then have $Q_{n} \geq Q_{n+1} \geq Q_{\infty}$, so that clearly $Q_{n} \rightarrow Q_{\infty}$ strongly as $n \rightarrow \infty$. Note

$$
Q_{n} \psi=\int_{K(n)} \alpha(g, x)^{1 / 2} \psi\left(g^{-1} x\right) d g
$$

Because $\pi$ is tame, by Kirillov's Lemma (2.3), we can find a nonzero $\psi \in L^{2}(X, \mu)^{K(\infty)}$ for some $m$ (recall that if $K(\infty)$ is a single factor, $K(\infty)_{m}$ is the stabilizer of the first $m$ basis elements; in general it is the corresponding product). We can assume $\psi$ is non-negative. By averaging $\psi$ over $K(m)$ we can then obtain a nonzero $K(m) \times K(\infty)_{m}$ invariant function $\phi$.

Now $K / K(m) \times K_{m}$ is a finite product of finite rank (infinite dimensional) Grassmannians (e.g., if $K=\mathrm{U}(H)$, the quotient is $\operatorname{Gr}(m, H)$, the space of $m$ dimensional subspaces of $H$ ). We can regard this space as symmetric space in the usual way.

In polar coordinates for our symmetric space the spherical function

$$
g \rightarrow\langle\phi, \pi(g) \phi\rangle=\int_{x} \alpha(g, x)^{1 / 2}|\phi(x)|^{2} d \mu(x)
$$

will depend only upon the radial variables, of which there is a finite number ( $m$ times the number of factors). Thus this function is completely determined by its restriction to the compact submanifold

$$
K(2 m) / K(m) \times K(2 m)_{m} \subseteq K / K(m) \times K_{m} .
$$

Thus we can find $\delta>0$ such that

$$
\int_{x} \alpha(g, x)^{1 / 2}|\phi|^{2}(x) d \mu(x)>\delta
$$


for all $g \in K(\infty)$. This estimate then clearly implies that $Q_{n} \phi$ converges to a nonzero limit. This concludes the proof.

We now describe some delimiting examples.

On $\mathbb{R}^{\infty}$ there are analogues of Lebesgue measure, i.e., measures which are invariant under translations by $\mathbb{R}_{0}^{\infty}$. These measures are infinite and $O(\infty)$ invariant. They are not equivalent to finite $O(\infty)$ invariant measures (see [Y]). Thus (4.1) fails in a disastrous way for $K(\infty)$ in the inductive limit topology.

A more subtle example is the following. First consider the action of $\mathrm{U}(\infty)$ on the finite rank Grassmannian $\operatorname{Gr}\left(n, \mathbb{C}^{\infty}\right)$. There is a unique invariant probability measure for this action (see [Pi1]). Thus a corollary of $(4.1)$ is that $\operatorname{Gr}\left(n, \mathbb{C}^{\infty}\right)$ carries a unique tame invariant measure class (this is true more generally for any finite rank flag space for $K(\infty))$.

We now try to pass to the infinite rank case. Consider the action of $\mathrm{U}(2 \infty)$ on the infinite Grassmannian $\mathrm{Gr}_{0}^{*}$ described in [Pi1] (this is a certain completion of $\operatorname{Gr}(\infty, 2 \infty)=\lim _{n} \operatorname{Gr}\left(n, \mathbb{C}^{2 n}\right)$ in the same way that $\operatorname{Gr}\left(n, \mathbb{C}^{\infty}\right)$ is a completion of $\left.\lim _{m \rightarrow \infty} \operatorname{Gr}\left(n, \mathbb{C}^{m}\right)\right)$. For this action there is a one parameter family of mutually disjoint quasiinvariant probabilities $\mu_{s}(s>-1)$, and there is a unique invariant probability, namely $\mu_{0}$. Thus in this case there is at least a continuum of invariant measure classes. This shows that it is not possible to relax our finite rank assumption in (4.1) for a general nonlinear action. I do not know of any counterexamples for linear actions however.

5. Invariant measures. If $G$ is a compact group, then Haar measure is a universal measure for $G$, in the sense that every invariant measure for $G$ is a convex combination of equivariant images of the left action of $G$ on Haar measure. We conjecture that the situation is approximately the same for tame actions of $K(\infty)$, in a sense which we will make precise at the end of this section. In the first part of this section we recall from [Pi3] the analogue of Haar measure for $K(\infty)$. We then consider various situations where it is possible to classify all of the invariant actions, in the process building up evidence for our conjectures at the end.

Since we are principally interested here in linear actions, we first note that in this context tameness is automatic.

(5.1) Proposition. If $\nu$ is a $K(\infty)$ invariant probability on $H(\pi)_{0}^{\wedge}$, where $\pi$ is a real tame action, then $\nu$ is automatically tame. 
Proof. The functions in $L^{2}(\nu)$ which are based on $H(\pi)_{0}^{K(\infty)_{n}}$ (i.e., of the form $\Phi=\Phi\left(\left\langle x_{j}, \xi\right\rangle\right)$ with $x_{j} K(\infty)_{n}$-invariant) and contained in $L^{2}(\nu)^{K(\infty)_{n}}$. Kirillov's criterion (2.3) for tameness implies that $\bigcup_{n} H(\pi)_{0}^{K(\infty)_{n}}$ is dense in $H(\pi)_{0}$, and functions depending on finitely many variables are dense in $L^{2}(\nu)$; hence $\bigcup_{n} L^{2}(\nu)^{K(\infty)_{n}}$ is dense in $L^{2}(\nu)$.

Let $\varepsilon_{1}, \ldots, \varepsilon_{n}, \ldots$ be an orthonormal basis for the real, complex, or quaternionic Hilbert space $H$. If $F$ denotes the scalar field (acting from the right), we then have a natural action

$$
\begin{gathered}
K(\infty) \times \mathscr{L}_{F}\left(\sum_{1}^{\infty} \varepsilon_{j} F, \prod_{1}^{\infty} \varepsilon_{j} F\right) \times K(\infty) \\
\rightarrow \mathscr{L}_{F}\left(\sum \varepsilon_{j} F, \prod \varepsilon_{j} F\right)
\end{gathered}
$$

given by composition, where $K(\infty)=\mathrm{SO}(\infty), \mathrm{U}(\infty)$, and $\mathrm{Sp}(\infty)$, respectively. For a product we concatenate these actions. Let $\nu_{G}$ denote the Gaussian measure on this linear space:

$$
d \nu_{G}(L)=\prod_{i, j}\left(\frac{1}{z} e^{-\left|L_{i \jmath}\right|^{2}} d m\left(L_{i j}\right)\right),
$$

which is an infinite product measure in the coordinates of the space $(m(\cdot)$ denotes Lebesgue measure and $z$ is a normalization constant). This measure is a good analogue of Haar measure for $K(\infty)$. One reason is the following Peter-Weyl type result.

(5.3) Proposition. The decomposition of $L^{2}\left(\nu_{G}\right)$ relative to the two sided action of $K(\infty)$ is

$$
L^{2}\left(\nu_{G}\right) \cong \sum \rho \times \rho^{*}
$$

where the sum is over all irreducible tame representations of $K(\infty)$.

The basic tools here are the Fourier transform and dual pair theory. If $V$ is a real Hilbert space with orthonormal baasis $v_{1}, v_{2}, \ldots$, then we have

$$
V_{0}=\sum \mathbb{R} v_{j} \subseteq V \subseteq V_{0}^{\wedge}=\prod \mathbb{R} v_{j}
$$

The Fourier transform (followed by some scaling that is not important to us) then defines an $O(\infty)$ equivariant map

$$
L^{2}\left(V_{0}^{\wedge}, \prod_{1}^{\infty} \frac{1}{\sqrt{2 \pi}} e^{-\left|\xi_{j}\right|^{2}} d \xi_{j}\right) \rightarrow S(V)^{\mathbb{C}}
$$


Thus if $F=\mathbb{R}$, as representations of $\mathrm{SO}(\infty) \times \mathrm{SO}(\infty)$,

$$
L^{2}\left(\nu_{G}\right) \cong S\left(\mathscr{L}_{2}(H)\right) \cong \sum \rho \times \rho .
$$

The decomposition of the symmetric algebra follows from dual pair theory as in finite dimensions.

$$
\begin{aligned}
& \text { If } \begin{array}{rl}
F & \mathbb{C} \\
L^{2}\left(\nu_{G}\right) & \cong S\left(\mathscr{L}_{2}(H) \mid \mathbb{R}\right)^{\mathbb{C}} \cong S\left(\mathscr{L}_{2}(H)\right) \otimes \bar{S}\left(\mathscr{L}_{2}(H)\right) \\
& \cong \sum \lambda^{*} \times \lambda \otimes \sum \eta \times \eta^{*} \cong \sum \lambda^{*} \otimes \eta \times \lambda \otimes \eta^{*} \cong \sum \rho \otimes \rho^{*}
\end{array}
\end{aligned}
$$

where the sums in $\lambda$ and $\eta$ are over the holomorphic tame representations of $\mathrm{U}(\infty)$, so that the last sum is over all tame representations.

If $F=\mathbb{H}$, viewing quaternionic linear operators as complex linear maps which are the +1 eigenspace of the operator $L \rightarrow j^{-1} \cdot L \cdot j$ (denoted by subscript + ),

$$
\begin{aligned}
L^{2}\left(\nu_{G}\right) & \cong S\left(\mathscr{L}_{2}\left(\left.H\right|_{\mathbb{C}}\right)_{+}\right) \otimes \bar{S}\left(\mathscr{L}_{2}\left(\left.H\right|_{\mathbb{C}}\right)_{+}\right) \\
& \cong S\left(\mathscr{L}_{2}\left(\left.H\right|_{\mathbb{C}}\right)_{+}\right) \otimes S\left(\mathscr{L}_{2}\left(\left.H\right|_{\mathbb{C}}\right)_{-}\right) \\
& \cong S\left(\mathscr{L}_{2}\left(\left.H\right|_{\mathbb{C}}\right)\right) \cong \sum \rho \otimes \rho
\end{aligned}
$$

where the sum is over all tame representations of $\operatorname{Sp}(\infty)$ (which are all self-dual).

The general case follows in an obvious way.

Another sense in which the Gaussian is a natural candidate for Haar measure is the following

(5.4) Proposition. The invariant probability distribution on the space $\sqrt{n} K(n)$ converges weakly to $\nu_{G}$. Moreover the convergence (of Fourier transforms) is uniform over finite dimensional spaces $\mathscr{L}\left(\sum_{1}^{N} \varepsilon_{j} F, \prod_{1}^{N} \varepsilon_{j} F\right)$.

This is proven in [Pi2] for $\mathrm{U}(\infty)$, the other cases following by essentially the same arguments.

We now want to take up the problem of classifying ergodic invariant probabilities for various actions of $K(\infty)$. We first consider

$$
K(\infty) \times \mathscr{L}\left(F^{n}, \prod_{1}^{\infty} \varepsilon_{j} F\right) \rightarrow \mathscr{L}\left(F^{n}, \prod_{1}^{\infty} \varepsilon_{j} F\right): L \rightarrow g \cdot L .
$$

For each $q \in \operatorname{Pos}\left(F^{n}\right)$, the space of positive semidefinite operators, the map $L \rightarrow L \cdot q$ is $K(\infty)$ equivariant. Let $q_{*} \nu_{G}$ denote the image. 
(5.6) Proposition. If $\mu$ is a $K(\infty)$-invariant probability for the action (5.5), then

$$
\mu=\int q_{*} \nu_{G} d \nu(q)
$$

where $\nu$ is a probability on $\overline{\operatorname{Pos}}\left(F^{n}\right)$ uniquely determined by $\mu$. In particular the $q_{*} \nu_{G}$ are the ergodic probabilities.

This is proven in [Pi2] using (5.3) and also in [Nes]. The striking fact is that the ergodic measures are parameterized by the orbits of $K$ acting on $\mathscr{L}\left(F^{n}, H\right)$, precisely as in finite dimensions.

This result, together with (3.5), has the following

(5.7) COROLlARY. The irreducible spherical functions for the rank $n$ Cartan motion group pair $\left((K(\infty) \times K(n)) \widetilde{\times} \mathscr{L}\left(F^{n}, H\right), K(\infty) \times\right.$ $K(n))$ are parameterized by the orbits of $K(n)$ in $\overline{\operatorname{Pos}}\left(F^{n}\right)$. If $x \in$ $\mathscr{L}\left(F^{n}, F^{\infty}\right)^{\wedge} \cong \mathscr{L}\left(\sum \varepsilon_{j} F, F^{n}\right)$ (via $\left.\mathbb{R e t r a c e}\right)$, and $x^{*} x$ has eigenvalues $\mu_{1}^{2}, \ldots, \mu_{n}^{2}$, the spherical corresponding to $\lambda=\operatorname{diag}\left(\lambda_{1}, \ldots, \lambda_{n}\right) \in$ $\overline{\operatorname{Pos}}\left(F^{n}\right)$ is given by

$$
\psi_{\lambda}(x)=\frac{\operatorname{det}\left(e^{-\lambda_{t}^{2} \mu_{j}^{2}}\right)}{\prod_{i<j}\left(\lambda_{i}^{2}-\lambda_{j}^{2}\right)\left(\mu_{i}^{2}-\mu_{j}^{2}\right)} .
$$

This parameterization is proven in [011] by different means.

By letting $n \rightarrow \infty$ in (5.6) it is easy to determine all of the ergodic probabilities for the left action of $K(\infty)$ in (5.2). The qualitative conclusion is the same: the ergodic actions are all equivariant images of $\nu_{G}$ (in fact they are of the form $q_{*} \nu_{G}$ (when properly interpreted), where $q$ is now an $\infty \times \infty$ nonnegative matrix (see $\S 3$ of [Pi2])).

We now take up the classification of ergodic probabilities for actions of the form $K(\infty) \times \mathfrak{p}(\infty)^{\wedge}$, where $\mathfrak{g}(\infty)=\mathfrak{k}(\infty) \oplus \mathfrak{p}(\infty)$ is the Cartan decomposition for a classical infinite rank symmetric pair of the noncompact type. It turns out that it suffices to work out one case,

$$
\mathfrak{g l}(\infty, \mathbb{C})=\mathfrak{u}(\infty) \oplus \operatorname{herm}(\infty)
$$

The basic fact is that if $\nu$ is a $\mathrm{U}(\infty)$ conjugation invariant probability on herm $(\infty)^{\wedge}$, then it is ergodic if and only if its Fourier transform has the form

$$
\phi(x)=\prod_{1}^{\infty} p\left(t_{j}\right)
$$

where $\left\{t_{j}\right\}=\operatorname{spectrum}(x), x \in \operatorname{herm}(\infty)$. More generally if we choose a maximal abelian subalgebra $\mathfrak{a}(\infty) \subseteq \mathfrak{p}(\infty)$ so that 
$\mathfrak{a}(n) \subseteq \mathfrak{p}(n)$ is maximal for all $n$, and we identify $\mathfrak{a}(\infty) \cong \sum \mathbb{R} \varepsilon_{j}$ by choosing an orthonormal basis $\varepsilon_{1}, \ldots, \varepsilon_{n}$ for $\mathfrak{a}(n)$, then we have

(5.8) LEMMA. If $\nu$ is a $K(\infty)$ invariant probability on $\mathfrak{p}(\infty)^{\wedge}$, then $\nu$ is ergodic if and only if its Fourier transform is of the form

$$
\phi(x)=\prod_{1}^{\infty} p\left(t_{j}\right)
$$

where $x$ is $K(\infty)$ conjugate to $\left(t_{j}\right) \in \mathfrak{a}(\infty)$.

This follows by the argument for the analogous fact about the irreducible spherical functions for $(G(\infty), K(\infty)$ ) (see $\S 3$ of [Pi4] or [Nes]).

(5.9) Proposition. The irreducible spherical functions for $(\mathrm{U}(\infty) \tilde{\times}$ $\operatorname{herm}(\infty), \mathrm{U}(\infty))$ are of the form $\phi(x)=\prod_{1}^{\infty} p\left(t_{j}\right)$,

$$
p(t)=e^{i \omega t} e^{-d t^{2}} \prod_{1}^{\infty}\left[\left(1+i d_{j} t\right) e^{-i d_{j} t}\right]^{-1}
$$

where spectrum $(x)=\left\{t_{j}\right\}, \omega, d$ and $d_{j}$ are real numbers, $d \geq 0$, and $\sum d_{j}^{2}<\infty$. This representation for $p$ is unique.

REMARK. It is interesting to note that we can write $\phi$ as

$$
\phi(x)=\exp \left(i \omega \operatorname{tr}(x)-d \operatorname{tr}\left(x^{2}\right)\right) \prod_{1}^{\infty} \operatorname{det}_{2}\left(1+i d_{j} x\right)^{-1}
$$

where $\operatorname{det}_{2}$ denotes the Hilbert-Schmidt regularized determinant. This shows that if $\omega=0$, then $\phi$ extends continuously to herm , the Hilbert-Schmidt hermitian operators (see (6.1) below).

Proof of (5.9). By multiplying $p(t)$ by $e^{-d t^{2}}$ if necessary, we can assume

$$
p(t)=\int_{-\infty}^{+\infty} e^{i \lambda t} f(\lambda) d \lambda
$$

where $f$ is analytic (and necessarily $f \geq 0, \int f=1$ ).

Because $x \rightarrow p\left(t_{1}\right) \cdots p\left(t_{n}\right)$ is a positive definite function on $\operatorname{herm}(n)$, it is a convex combination of the irreducible spherical functions for $(\mathrm{U}(n) \tilde{\times} \operatorname{herm}(n), \mathrm{U}(n))$. Our first task is to determine the density in terms of $f$. 
The irreducible spherical functions are parameterized by the orbits of $\mathrm{U}(n)$ in $\operatorname{herm}(n)^{\wedge}$. Because our pair corresponds to a complex group, they have a simple form: if $\lambda=\operatorname{diag}\left(\lambda_{1}, \ldots, \lambda_{n}\right)$, then

$$
\psi_{\lambda}(t)=\int_{\mathrm{U}(n)} e^{-i t r \lambda k t k^{-1}} d k=\frac{\operatorname{det}\left(e^{i \lambda_{i} t_{j}}\right)}{\prod_{i<j} i\left(\lambda_{i}-\lambda_{j}\right)\left(t_{i}-t_{j}\right)} .
$$

It follows that we have

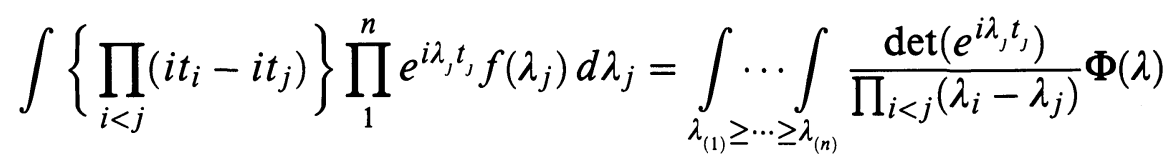

where $\Phi$ is a probability measure. The left hand side equals

$$
\begin{aligned}
& \int\left\{\operatorname{det}\left(\left(-\partial_{j}\right)^{i-1}\right) \prod e^{i \lambda_{j} t_{j}}\right\} \prod f\left(\lambda_{j}\right) d \lambda_{j} \\
& =\int\left(\prod e^{i \lambda_{j} t_{j}}\right)\left\{\operatorname{det}\left(\partial_{j}\right)^{i-1} \prod f\left(\lambda_{j}\right)\right\} \prod d \lambda_{j} \\
& =\sum_{\sigma \in \operatorname{perm}(n)} \int \cdots \int_{\lambda_{\sigma(1)} \geq \cdots \geq \lambda_{\sigma(n)}}\left(\prod e^{i \lambda_{j} t_{j}}\right) \operatorname{det}\left(f^{(i-1)}\left(\lambda_{j}\right)\right) \\
& =\int_{\lambda_{(1)} \geq \cdots \geq \lambda_{(n)}} \ldots \int_{\operatorname{det}\left(e^{i \lambda_{i} t_{j}}\right) \operatorname{det}\left(f^{(i-1)}\left(\lambda_{j}\right)\right) \prod d \lambda_{j}}
\end{aligned}
$$

This determines the density for $\Phi$.

We now have determined the conditions for $\Pi_{1}^{\infty} p\left(t_{j}\right)$ to represent an irreducible spherical representation:

$$
\operatorname{det}\left(f^{(i-1)}\left(\lambda_{j}\right)\right)_{1 \leq i, j \leq n} \geq 0, \quad \lambda_{1}>\lambda_{2}>\cdots>\lambda_{n},
$$

for all $n$. Since $f$ is analytic we actually have strict inequality except at a discrete collection of points.

We can now apply basic results of the theory of total positivity. The equation (5.10) says that the kernel $K(x, y)=f(x-y)$ is extended totally positive (on intervals where we have strict inequality). Theorem (2.5) of [Kar] asserts that $K$ is actually totally positive. Schoenberg's representation Theorem ((a) of (3.2) in [Kar]) now completes the proof.

To show how this classification can be extended to the other classical infinite rank pairs, we observe that there is a maze of relations among 
them, some of which are indicated in the following scheme: (5.11)

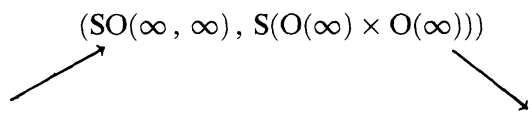

$(\mathrm{GL}(\infty, \mathbb{R}), \mathrm{O}(\infty)) \rightarrow(\mathrm{Sp}(\infty, \mathbb{R}), \mathrm{U}(\infty)) \rightarrow(\mathrm{U}(\infty, \infty), \mathrm{U}(\infty) \times \mathrm{U}(\infty)) \rightarrow\left(\mathrm{SO}^{*}(4 \infty), \mathrm{U}(2 \infty)\right)$
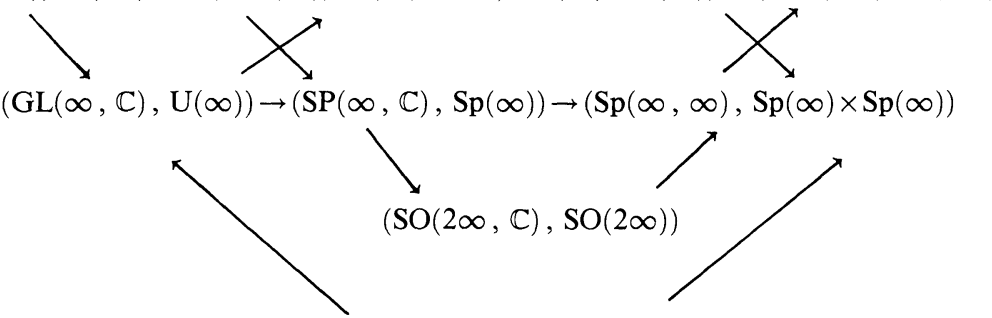

$(\mathrm{GL}(\infty \mathbb{H}), \mathrm{Sp}(\infty))$.

Each arrow represents an injective map of symmetric pairs with the property that it is an isomorphism of the radial subgroups $\exp (\mathfrak{a}(\infty))$. The mappings we have indicated are dual to the ones constructed in $\S 3$ of [Pi4]. It follows from (5.8) that for each of the corresponding maps of Cartan motion groups, the pullback defines an injection on the set of irreducible spherical functions for $(K(\infty) \tilde{\times} \mathfrak{p}(\infty), K(\infty))$. Thus $\mathrm{O}(\infty, \mathbb{R}) \tilde{\times} \operatorname{Sym}(\infty, \mathbb{R})$ is a sort of universal case. To illustrate how these maps are used, we consider this case next.

(5.12) Proposition. The irreducible spherical functions for $(\mathrm{O}(\infty, \mathbb{R})$ $\widetilde{x} \operatorname{Sym}(\infty), \phi(\infty))$ are of the form $\phi(x)=\prod_{1}^{\infty} p\left(t_{j}\right)$,

$$
p(t)=e^{i \omega t} e^{-d t^{2}} \prod_{1}^{\infty}\left[\left(1+i d_{j} t\right) e^{-i d_{J} t}\right]^{-1 / 2}
$$

where the parameters are precisely as in (5.9).

Proof. Consider the natural maps

$$
\begin{aligned}
\mathrm{O}(\infty) & \tilde{\times} \operatorname{Sym}(\infty, \mathbb{R}) \rightarrow \mathrm{U}(\infty) \tilde{\times} \operatorname{herm}(\infty, \mathbb{C}) \\
\rightarrow & \mathrm{O}(2 \infty) \tilde{\times} \operatorname{Sym}(2 \infty, \mathbb{R})
\end{aligned}
$$

where the first map is given by $\mathbb{C}$ linear extension of operators, the second by reduction of the scalar field. Now suppose $\phi=\prod p\left(t_{j}\right)$ is an irreducible spherical function for $\mathrm{O}(\infty) \tilde{\times} \operatorname{Sym}(\infty, \mathbb{R})$. If $\Phi$ is the spherical function for $\mathrm{O}(2 \infty) \tilde{\times} \operatorname{Sym}(2 \infty, \mathbb{R})$ corresponding to $p(t)$, then relative to the mappings $(5.13)$

$$
\Phi\left(\begin{array}{ll}
x & \\
& x
\end{array}\right)=\phi(x)^{2} \quad \text { for } x \in \operatorname{Sym}(\infty, \mathbb{R}) \text {. }
$$


This shows that $\phi^{2}$ extends uniquely to an irreducible spherical function for $\mathrm{U}(\infty) \tilde{\times}$ herm $(\infty)$.

To conclude the proof of (5.12) it suffices to show that each $\phi(x)$ in (5.12) does define a positive definite function on $\operatorname{Sym}(\infty, \mathbb{R})$. It suffices to consider $p(t)=\prod_{1}^{N}\left(1+d_{j} t\right)^{1 / 2}$, for the others are limits of these.

Let $d=\operatorname{diag}\left(d_{1}, \ldots, d_{N}, 0, \ldots\right)$ and consider the $\mathrm{O}(\infty)$-equivariant map

$$
\mathscr{L}\left(\sum \mathbb{R} \varepsilon_{j}, \prod \mathbb{R} \varepsilon_{j}\right) \rightarrow \operatorname{Sym}(\infty, \mathbb{R})^{\wedge}: L \rightarrow L \cdot d \cdot L^{*} .
$$

The image of $\nu_{G}$ under this map is ergodic, and it is easy to check that the Fourier transform $\phi=\prod p\left(t_{j}\right)$, with $p$ as above.

Now that the "universal case" is in hand, it is not difficult to use the maze (and various ad hoc arguments) to classify the ergodic probabilities in all cases. We will not pursue this beyond remarking that in all cases, modulo characters and the Gaussian, there is a precise correspondence with the orbits of $K$ in $\mathfrak{p}_{2}$, and stating the result for the action (5.2) (this will be needed elsewhere).

(5.14) Proposition. The ergodic probabilities for the action (5.2) have Fourier transform of the form $\nu(x)=\prod p\left(u_{j}\right)$, where

$$
p(u)=e^{-d u^{2}} \prod_{1}^{\infty}\left(1+d_{j}^{2} u^{2}\right)^{-k / 2},
$$

$\operatorname{spec}\left(x^{*} x\right)=\left\{u_{j}^{2}\right\}, d \geq 0, \sum d_{j}^{2}<\infty$, and $k=1,2$ and 4 for $F=\mathbb{R}, \mathbb{C}$ and $\mathbb{H}$, respectively.

We now turn to the general case, $K(\infty) \tilde{x} H(\pi)_{0}$, where $\pi$ is a tame real orthogonal representation. Let $\beta$ denote the space of ergodic $K(\infty)$-invariant probabilities on $H(\pi)_{0}^{\wedge}$ with the weak*-topology (which we can identify with the irreducible spherical functions of $\left(K(\infty) \tilde{\times} H(\pi)_{0}, K(\infty)\right)$ with the topology of convergence on compact sets of $H(\pi)_{0}$ (with the inductive limit topology).

(5.15) Proposition. $\beta$ is a topological abelian semigroup with identity and involution, where the operation is convolution and the involution is conjugation.

Note that in terms of the Fourier transform, the operation is simply multiplication of irreducible spherical functions. 
To establish this we note that

$$
H(\pi \otimes \eta)^{K}=H(\pi)^{K} \otimes H(\eta)^{K}
$$

for tame representations $\pi$ and $\eta$ of $K$, which follows from the discreteness of the spectrum and the infinite dimensionality of all nontrivial irreducible tame representations. Therefore if $\pi$ and $\eta$ are irreducible spherical, (5.16) shows that $\pi \otimes \eta$ contains a unique irreducible spherical summand. Therefore the product of the spherical functions is still irreducible.

There is a simple way to generate measures in $\beta$. We first consider a specific example, the action of $\mathrm{U}(H)$ on $\Lambda^{n} H$, the $n$th exterior power. If $H$ were finite dimensional, then the ergodic invariant probabilities would simply be obtained by smearing Haar measure over each of the orbits. To obtain ergodic $\mathrm{U}(\infty)$-invariant probabilities in $\Lambda^{n}\left(\mathbb{C}^{\infty}\right)$, we consider equivariant images of the surrogate Haar measure for $\mathrm{U}(\infty)$ : for each point $\xi_{0} \in \Lambda^{n}\left(\sum \mathbb{C} \varepsilon_{j}\right)$, the map $\mathscr{L}\left(\sum \mathbb{C} \varepsilon_{j}, \Pi \mathbb{C} \varepsilon_{j}\right) \rightarrow \Lambda^{n}\left(\mathbb{C}^{\infty}\right): L \rightarrow L \cdot \xi_{0}$ is clearly well-defined and $\mathrm{U}(\infty)$ equivariant; the image of the Gaussian below (5.2) is then an ergodic invariant probability (we choose this example partly because in [Ver] it is claimed that the linear Gaussians are the only ergodic probabilities in $\Lambda^{n} \mathbb{C}_{\infty}$, whereas these nonlinear images of Gaussians show that there exist many other examples).

In the general case of $K(\infty)$ acting on $H(\pi)_{0} \subseteq H(\pi) \subseteq H(\pi)_{0}^{\wedge}$, we can consider equivariant images of our surrogate Haar measure corresponding to different points of $\xi_{0} \in H(\pi)_{0}$. A fairly safe conjecture is that as we vary $\xi_{0}$ we obtain a dense subset of $\beta$ modulo characters. This is true for all the Cartan motion actions. For example for $\mathrm{U}(\infty) \tilde{\times} \operatorname{herm}(\infty)$, the "orbit measures" through points in herm $(\infty)$ are those with Fourier transform of the form

$$
\hat{\nu}(x)=\prod_{i=1}^{\infty} \prod_{j=1}^{n}\left(1+d_{j} t_{i}\right)^{-1}
$$

in (5.9) and the classification shows that these are dense (the Gaussian is a limit of these because $\left.\exp \left(-d t^{2}\right)=\lim \left|1+i\left(\frac{d}{n}\right)^{1 / 2} t\right|^{-2 n}\right)$.

A more difficult question is whether all ergodic probabilities for $K(\infty)$ on $H(\pi)_{0}^{\wedge}$ can somehow be viewed as equivariant images of Gaussians. Some motivation for viewing measures as equivariant images of canonical measures is provided by $\S 7$.

6. Some qualitative consequences. Suppose $\pi$ is a tame real orthogonal action. Suppose $\nu$ is an ergodic $K(\infty)$-invariant probability on 
$H(\pi)_{0}^{\wedge}$, and consider the natural representation $T$ :

$$
T: K(\infty) \tilde{x} H(\pi)_{0} \rightarrow \mathrm{U}\left(L_{2}(\nu)\right) .
$$

The constant function $1 \in L^{2}(\nu)$ induces a mapping

$$
K(\infty) \tilde{x} H(\pi)_{0} / K(\infty) \cong H(\pi)_{0} \rightarrow \mathbb{P}\left(L^{2}\right):(x, g) \rightarrow \mathbb{P}(T(x, g) \cdot 1) .
$$

Assuming this map is smooth, it is natural to pull back the FubiniStudy metric and compare this with the metric for $H(\pi)$. In irreducible situations these should be infinitesimally equivalent. When this is the case the continuity properties of $T$ are sharply constrained. Because of the results in the previous section, we can apply this idea to Cartan motion groups.

Let $(\mathfrak{g}(\infty), \mathfrak{k}(\infty))$ be a classical noncompact type infinite rank symmetric pair. For each $1 \leq q \leq \infty$, we can complete $\mathfrak{p}(\infty)$ in the Schatten $q$-class to obtain a Banach space $\mathfrak{p}_{q}$.

(6.1) Proposition. Suppose $\rho$ is an admissible unitary representation for $K(\infty) \tilde{x} \mathfrak{p}(\infty)$.

(a) if $\rho$ extends continuously to $K(\infty) \tilde{x}_{p_{q}}$ for some $q>2$, then $\rho$ vanishes on $\mathfrak{p}(\infty)$, so that $\rho$ is simply a tame representation of $K(\infty)$.

(b) $\rho$ always extends uniquely to a continuous representation of $K \widetilde{\times} \mathfrak{p}_{1}$.

(c) If $\rho$ corresponds to the probability measures $\left\{\nu_{m}\right\}$ as in (3.4), and if $\hat{\nu}_{m}$ is real for each $m$, then $\rho$ extends uniquely to a continuous representation of $K \widetilde{\times} \mathfrak{p}_{2}$.

By (4.1) we can assume the $\nu_{m}$ are invariant probability measures. It suffices to consider the case when $\rho$ is spherical, since the cocycle plays no role. One way to prove (6.1) is then to observe that the arguments in $\S 6$ of [Pi4] apply without change to Cartan motion groups (as opposed to the symmetric pairs themselves). Another is simply to inspect the formulas of the previous section for the irreducible spherical functions.

I expect that this result actually holds for all actions $\pi$ having a finite number of irreducible components. This would follow from the arguments in [Pi4] if we knew that every measure $\nu \in \beta(\pi)$ had a Fourier transform which is smooth on $H(\pi)_{0}$.

Part (c) of (6.1) shows for example that every separable unitary representation for the semidirect product $\left(\mathrm{U}\left(H_{+}\right) \times \mathrm{U}\left(H_{-}\right)\right) \widetilde{\times} \mathscr{L}_{1}\left(H_{+}, H_{-}\right)$ has a continuous extension to $\left(\mathrm{U}\left(H_{+}\right) \times \mathrm{U}\left(H_{-}\right)\right) \widetilde{\times} \mathscr{L}_{2}\left(H_{+}, H_{-}\right)$. This 
is used in [Pi5] to illuminate certain renormalization processes in field theory.

7. Examples of cocycles. For a semidirect product $K \widetilde{\times} A$ with $K$ compact, the $K$-ergodic measure classes in $A^{\wedge}$ correspond simply to $K$-orbits, and for a $K$-orbit $K \cdot \chi \subseteq A^{\wedge}$, the cocycle cohomology is equivalent to the representation theory of the stability subgroup $K_{\chi}-$ the equivalence is gotten by assigning to a representation $\pi$ of $K_{\chi}$ the $L^{2}$ sections of $K \times_{\pi} H(\pi)$, the bundle over the orbit $K \cdot \chi$ induced by the representation.

Now consider one of our semidirect products $K(\infty) \tilde{\times} H(\pi)_{0}$, and supose $\nu$ is an ergodic $K(\infty)$-invariant probability on $H(\pi)_{0}^{\wedge}$. By example we will try to argue that one should compute the cocycle cohomology in the following way. To compute $H^{1}\left(K, F_{\nu}\left(H(\pi)_{0}^{\wedge}, \mathrm{U}\left(\mathscr{H}_{n}\right)\right)\right)$ for $n<\infty$, we should proceed as in finite dimensions: realize $\nu$ as an equivariant image of a linear action on a Gaussian (the substitute for Haar measure) and thereby determine the virtual stabilizer subgroup; the $n$-dimensional representations of this virtual subgroup should then exhaust the cohomology. To compute $H^{1}\left(K, F_{\nu}\left(H(\pi)_{0}^{\wedge}, \mathrm{U}\left(\mathscr{C}_{\infty}\right)\right)\right)$, we look the other direction-we determine the equivariant image of $\nu$ and pull the cocycles back. In particular since we can always push $\nu$ to a point mass, we are claiming that the infinite cohomology is never zero. This is the content of (7.1) below.

Let $\operatorname{Hom}(G ; A)$ denote the set of equivalence classes of continuous homomorphisms from $G$ to $A$, when $G$ and $A$ are topological groups.

(7.1) Proposition. Let $\nu$ be an ergodic $K(\infty)$-invariant probability on $H(\pi)_{0}^{\wedge}$. The natural map

$$
\operatorname{Hom}\left(K ; \mathrm{U}\left(\mathscr{H}_{\infty}\right)\right) \rightarrow H^{1}\left(K, F_{\nu}\left(H(\pi)_{0}^{\wedge}, \mathrm{U}\left(\mathscr{H}_{\infty}\right)\right)\right)
$$

(where we regard a homomorphism as a constant function on $H(\pi)_{0}^{\wedge}$ ) is injective.

Proof. This is equivalent to showing that if $\rho$ is an irreducible tame representation for $K(\infty)$, then $T_{\nu} \otimes \rho$ is irreducible for $K(\infty) \tilde{\times} H(\pi) \bar{\theta}$, where $T_{\nu}$ is the irreducible spherical representation on $H\left(T_{\nu}\right)=$ $L^{2}\left(H(\pi)_{0}^{\wedge}, \nu\right)$ corresponding to $\nu$.

Consider the decomposition

$$
\left.T_{\nu}\right|_{K(\infty)}=\mathbb{C} 1 \oplus(\mathbb{C} 1)^{\perp} .
$$


All the irreducible pieces in $(\mathbb{C} 1)^{\perp}$ have degree $>0$. Thus by $(2.6)$

$$
\left.T_{\nu} \otimes \rho\right|_{K(\infty)}=H(\rho) \oplus H(\rho)^{\perp}
$$

where all the irreducible pieces in $H(\rho)^{\perp}$ have degree $>$ degree $(\rho)$. Thus $\rho$ has multiplicity one.

Since the constants $H(\rho) \subseteq L^{2}(\nu) \otimes H(\rho)$ generate the representation space for $K(\infty) \tilde{\times} H(\pi)_{0}$, it follows that $T_{\nu} \otimes \rho$ is irreducible.

We next consider a measure which has only a point as equivariant image. Consider the Cartan motion group $\mathrm{U}(\infty) \widetilde{\times}$ herm $(\infty)$ and the "orbit measure" through $\sum_{1}^{n} d \varepsilon_{j} \otimes \varepsilon_{j}^{*}=\operatorname{diag}(d, \ldots, d, 0, \ldots)$ (in the sense of $\S 5$ ). The "orbit" in this case will (measure theoretically) turn out to be a Grassmannian (if we considered the "orbit measure" through a general point $\sum_{1}^{\infty} d_{j} \varepsilon_{j} \otimes \varepsilon_{j}^{*}$, we would obtain a flag manifold).

We can take $d=1$. To describe the map that gives the "orbit measure", for $L \in \mathscr{L}\left(\sum \mathbb{C} \varepsilon_{j}, \Pi \mathbb{C} \varepsilon_{j}\right)$ write $L=\left(\begin{array}{c}\alpha \beta \\ \gamma \delta\end{array}\right)$, where $\alpha$ is $n \times n$, etc. The map is then

$$
\mathscr{L} \rightarrow \operatorname{herm}(\infty)^{\wedge}: L \rightarrow L \operatorname{diag}(1, \ldots, 1,0, \ldots) L^{*}
$$

or

$$
\left(\begin{array}{ll}
\alpha & \beta \\
\gamma & \delta
\end{array}\right) \rightarrow\left(\begin{array}{cc}
\alpha \alpha^{*} & \alpha \gamma^{*} \\
\gamma \alpha^{*} & 0
\end{array}\right)
$$

This map does not depend upon $\beta$ and $\delta$, so by throwing away a set of measure zero we can view the "orbit" as the image of

(7.2) $\mathscr{L}\left(\mathbb{C}^{n}, \prod \mathbb{C} \varepsilon_{j}\right) / \mathrm{U}(n) \rightarrow \operatorname{herm}(\infty)^{\wedge}:\left(\begin{array}{l}\alpha \\ \gamma\end{array}\right) \rightarrow\left(\begin{array}{cc}\alpha \alpha^{*} & \alpha \gamma^{*} \\ \gamma \alpha^{*} & 0\end{array}\right)$

where the prime means we consider nonsingular maps only, and we have cancelled out the right $\mathrm{U}(n)$ action $k:\left(\begin{array}{l}\alpha \\ \gamma\end{array}\right) \rightarrow\left(\begin{array}{c}\alpha k \\ \gamma k\end{array}\right)$. A basic fact is that the map

$$
\mathscr{L}\left(\mathbb{C}, \prod \mathbb{C} \varepsilon_{j}\right) / \mathrm{U}(n) \rightarrow \mathscr{L}\left(\mathbb{C}^{n}, \prod \mathbb{C} \varepsilon_{j}\right)^{\prime} / \mathrm{GL}(n) \cong \mathrm{Gr}\left(n, \mathbb{C}^{\infty}\right)
$$

induces a measure algebra isomorphism, where both spaces are equipped with the image of the Gaussian (see (3.8) of [Pi3]). Thus what we are considering is the cohomology for $\mathrm{U}(\infty)$ acting on $\operatorname{Gr}\left(n, \mathbb{C}^{\infty}\right)$ relative to the unique tame invariant measure class. We should therefore expect to obtain "induced" cohomology from representations of $\mathrm{U}(n) \times \mathrm{U}(H)_{n}$, in addition to the cohomology pulled back from a point (by (7.1)). 
Since in (7.2) we have described the "orbit" as a quotient of the $\mathrm{U}(n)$ principal bundle $\mathscr{L}^{\prime}=\mathscr{L}^{\prime}\left(\mathbb{C}^{n}, \prod_{1}^{\infty} \mathbb{C} \varepsilon_{j}\right)$, it is clear that there is an injection of the $\mathrm{U}(n)$ representation theory (this is finite rank cohomology coming from viewing our measure as an image of a Gaussian). Moreover there is clearly a version of Frobenius reciprocity. If $\pi$ is a representation for $\mathrm{U}(n)$, then the induced module for $\mathrm{U}(\infty)$ is given by

$$
L^{2}\left(\mathscr{L}^{\prime} \times_{\pi} H(\pi)\right) \cong\left(L^{2}\left(\mathscr{L}^{\prime}, \nu_{G}\right) \otimes H(\pi)\right)^{\mathrm{U}(n)} .
$$

When $\pi$ is irreducible this leads to

$$
L^{2}\left(\mathscr{L}^{\prime} \times_{\pi} H(\pi)\right) \cong \sum m\left(\rho^{*} \otimes \eta\right) \rho^{*} \otimes \eta,
$$

where the sum is over all irreducible tame representations of $U(\infty)$ (which are of the form $\rho^{*} \otimes \eta, \rho$ and $\eta$ being holomorphic), and the multiplicity is given by the usual reciprocity formula

$$
m\left(\rho^{*} \otimes \eta\right)=m\left(\pi \times 1, \rho^{*} \otimes \eta \mid \mathrm{U}(n) \times \mathrm{U}(\infty)_{n}\right) .
$$

For we have

$$
L^{2}\left(\mathscr{L}\left(\sum \mathbb{C} \varepsilon_{j}, \prod \mathbb{C} \varepsilon_{j}\right), \nu_{G}\right)^{\mathrm{U}(\infty)_{n}}=L^{2}\left(\mathscr{L}\left(\mathbb{C}^{n}, \prod \mathbb{C} \varepsilon_{j}\right), \nu_{G}\right)
$$

where $\mathrm{U}(\infty)_{n}$ is acting on the right of $\mathscr{L}\left(\sum \mathbb{C} \varepsilon_{j}, \Pi \mathbb{C} \varepsilon_{j}\right)$. By (5.3) with $F=\mathbb{C}$ it follows that

$$
L^{2}\left(\mathscr{L}^{\prime}, \nu_{G}\right) \cong \sum \rho^{*} \otimes \eta \times\left(\rho \otimes \eta^{*}\right)^{\mathrm{U}(\infty)_{n}} .
$$

Inserting this into (7.3) then yields (7.5).

Since the representations of $\mathrm{U}(H)_{n}$ are always infinite dimensional (if nontrivial), as are the cocycles provided by (7.1), it seems reasonable to insert the following

\section{(7.6) Conjecture. The map}

$$
\operatorname{Hom}\left(\mathrm{U}(n) \times \mathrm{U}(H)_{n}, \mathrm{U}(N)\right) \rightarrow H^{1}\left(\mathrm{U}(H), F\left(\operatorname{Gr}\left(n, \mathbb{C}^{\infty}\right), \mathrm{U}(N)\right)\right.
$$

defined by the induction process above (which we know is injective) is an isomorphism.

Note that all this cohomology vanishes when pulled back to $\mathscr{L}^{\prime}$. We will see that this is not so for the infinite dimensional induced representations we will now construct.

To induce a general time representation of $\mathrm{U}(n) \times \mathrm{U}(\infty)_{n}$, it suffices (as we will see below) to consider $1 \times H_{-}$, where $H_{-}=\overline{\operatorname{span}}\left\{\varepsilon_{j}: j>n\right\}$ is the defining representation for $\mathrm{U}(\infty)_{n}$. Intuitively this should be 
the $L^{2}$ sections of the bundle $\mathrm{U}(H) \times_{\mathrm{U}(n) \times \mathrm{U}\left(H_{-}\right)} H_{-} \rightarrow \mathrm{Gr}(n, H)$. The problem is that the measure is not supported on $\operatorname{Gr}(n, H)$. It turns out that this bundle extends in a $\mathrm{U}(\infty)$-equivariant manner to a Hilbert bundle over the space $\operatorname{Gr}\left(n, \mathbb{C}^{\infty}\right)$. We will check this by observing that the cocycle extends.

For this purpose we consider graph coordinates at the basepoint $\mathbb{C}^{n} \in \operatorname{Gr}(n, H)$ :

$$
\mathscr{L}\left(\mathbb{C}^{n}, H_{-}\right) \rightarrow \operatorname{Gr}(n, H): z \rightarrow \operatorname{graph}(z) .
$$

A (carefully chosen) unitary trivialization over this coordinate patch is then given by the map

$$
\begin{gathered}
\mathscr{L}\left(\mathbb{C}^{n}, H_{-}\right) \times H_{-} \rightarrow \mathrm{U}(H) \times \mathrm{U}(n) \times \mathrm{U}\left(H_{-}\right) H_{-} \\
(z, v) \rightarrow\left[\left(\begin{array}{cc}
x_{1} & -z^{*} y \\
z x_{1} & y
\end{array}\right), v\right],
\end{gathered}
$$

where the square brackets denote equivalence classes of pairs, and $x_{1}=x_{1}(z), y=y(z)$ are defined by the following criteria:

$$
x_{1}^{-1^{*}} x_{1}^{-1}=1+z^{*} z, \quad y^{-1^{*}} y^{-1}=1+z z^{*},
$$

$y: H_{-} \rightarrow H_{-}$has positive diagonal entries and is triangular, i.e., $y: H_{-} \rightarrow H_{-}$has positive diagonal entries and is triangular, i.e., $y\left(\sum_{n+1}^{M} \mathbb{C} \varepsilon_{j}\right) \subseteq \sum_{n+1}^{M} \mathbb{C} \varepsilon_{j}$ for all $M$ ( $x_{1}$ can be defined in a similar manner, but this is not so important). We claim that the action of $g=\left(\begin{array}{ll}a & b \\ c & d\end{array}\right) \in \mathrm{U}(\infty)$ is given by

$$
\begin{aligned}
& z \rightarrow g \cdot z=(c+d z)(a+b z)^{-1}, \\
& v \rightarrow g \cdot v=d(g, g \cdot z) v
\end{aligned}
$$

where

$$
d(g, g \cdot z)=y(g \cdot z)^{-1}\left(d(g)-c(g) z^{*}\right) y(z) .
$$

It will follow from this that the action on sections, in these coordinates, is given by

$$
(g \cdot \beta)(z)=d(g, z) \beta\left(g^{-1} \cdot z\right) .
$$

This calculation is of course straightforward, but there is an important observation to be made. If $g \in \mathrm{U}(n+m) \subseteq \mathrm{U}(\infty)$, the matrix of $d(g)-c(g) z^{*}$, relative to splitting the basis of $H_{-}$into $\left\{\varepsilon_{j}: n<j \leq\right.$ $n+m\}$ and $\left\{\varepsilon_{j}: n+m<j\right\}$, is of the form

$$
d-c z^{*}=\left(\begin{array}{cc}
* & * \\
0 & 1
\end{array}\right) \text {. }
$$


When we multiply this on the right by $y(z)$, we obtain a matrix which is upper triangular except in the upper $m \times m$ block, thus $d(g, g \cdot z)^{-1}$, which is the unitary matrix that brings $\left(d-c z^{*}\right) y$ back into the upper triangular form $y(g \cdot z)$, is of the form $1+$ finite rank. Furthermore, $d(g, g \cdot z)$ only depends upon a finite number $\left(m^{2}\right)$ of the entries of $z$. For all the calculations are "local": the upper $m \times m$ block of $d-c z^{*}$ depends upon finitely many $z_{i j}$, the entries in $y$ depend upon finitely many $z_{i j}$, since $y$ is obtained by Gaussian elimination and inverting a triangular matrix, etc. This essentially proves the following

(7.10) Lemma. For $g \in \mathrm{U}(\infty)$ the cocycle $d(g, z)$ defines a class in $H^{1}\left(\mathrm{U}(H), F\left(\mathrm{Gr}\left(n, C^{\infty}\right), \mathrm{U}\left(H_{-}\right)\right)\right.$) (relative to the unique tame invariant measure class).

The only remaining point to check is that the representation corresponding to $d(g, z)$ is tame (this implies the extension from $\mathrm{U}(\infty)$ to $\mathrm{U}(H))$. This follows from noting that for $g=\left(\begin{array}{c}{ }^{a} \\ d\end{array}\right), d(g, z) \equiv d$, and using Kirillov's Lemma (2.3).

We will denote the tame representation of $\mathrm{U}(H)$ corresponding to $d(g, z)$ by $\operatorname{Ind}\left(1 \times H_{-}\right)$, the "representation induced by $1 \times H_{-}$ of $\mathrm{U}(n) \times \mathrm{U}\left(H_{-}\right)$". For an arbitrary irreducible tame representation $\rho^{*} \otimes \eta$ of $\mathrm{U}\left(H_{-}\right)$, the induced representation corresponding to $1 \times \rho^{*} \otimes \eta$ is defined the cocycle

$$
\rho^{*}(d(g, z)) \otimes \eta(d(g, z)) .
$$

Combining this with the inducing mechanism which we described for $\mathrm{U}(n)$ earlier, we obtain a map

$\operatorname{Hom}\left(\mathrm{U}(n) \times \mathrm{U}\left(H_{-}\right), \mathrm{U}\left(\mathscr{H}_{\infty}\right)\right) \rightarrow H^{1}\left(\mathrm{U}(H), F\left(\operatorname{Gr}\left(n, C^{\infty}\right), \mathrm{U}\left(\mathscr{H}_{\infty}\right)\right)\right)$,

where for irreducible representations

$$
\pi \times \rho^{*} \otimes \eta \rightarrow \operatorname{Ind}\left(\pi \times \rho^{*} \otimes \eta\right) .
$$

These representations cannot be decomposed by the standard kind of argument we employed for $\operatorname{Ind}(\pi \times 1)$ above. It is natural to suspect that the standard reciprocity formula holds, but I have made little headway in confirming this.

There are two remarks which should be added. The first is that these cocycles do arise naturally, despite their mysterious appearance 
here. If one considers the action of $\mathrm{U}(\infty)$ in the cotangent bundle of $\operatorname{Gr}\left(n, C^{\infty}\right)$, it is possible to define an invariant inner product in the fibers by a renormalization process ([Pi6]). The cocycle that arises is one of those appearing here. This renormalization process yields a Laplace operator, and decomposing the representation corresponding to the cocycle would decompose the operator.

The second remark is that these cocycles do not simplify when pulled back to $\mathscr{L}^{\prime}$. This is easily verified by restricting the corresponding representation to $\mathrm{U}(n) \times \mathrm{U}\left(H_{-}\right)$. Thus the classification problem is quite complicated even for elemental actions such as

$$
\mathrm{U}(\infty) \times\left(C^{\infty}, \text { Gaussian measure }\right)
$$

\section{REFERENCES}

[Bo] R. Boyer, Representations of the Hilbert Lie group $\mathrm{U}(H)_{2}$, Duke Math. J., 47, no. 2, (1980), 325-344.

[Kar] S. Karlan, Total Positivity, Stanford University Press, Stanford, CA 1968.

[Kir] A. A. Kirillov, Representations of the infinite dimensional unitary group, Dokl. Akad. Nauk USSR, 212, No. 2 (1973), 288-290.

[Mo] C. Moore, Group extensions and cohomology for locally compact groups. III, Trans. Amer. Math. Soc., 221, no. 1, (1976), 1-33.

[Nes] N. Nessonov, A complete classification of the representations of $\mathrm{GL}(\infty)$ containing the identity representation of the unitary subgroup, Math. USSR Sbornik, 5, no. 1 (1987), 122-147.

[O11] G. I. Ol'shanskii, Unitary representations of the infinite dimensional classical groups $\mathrm{U}(p, \infty), \mathrm{SO}_{0}(p, \infty), \mathrm{Sp}(\infty)$ and the corresponding motion groups, Soviet Math. Dokl., 19 (1983).

[O12] __ Infinite dimensional groups of finite $\mathbb{R}$-rank: description of representations and asymptotic theory, Funct. Anal. Appl., 18, no. 1 (1984), 22-34.

[O13] _- Unitary representations of the group $\mathrm{SO}_{0}(\infty, \infty)$ as limits of unitary representations of the groups $\mathrm{SO}_{0}(\eta, \infty)$ as $\eta \rightarrow \infty$, preprint.

[Pi1] D. Pickrell, Measures on infinite-dimensional Grassmann manifolds, J. Funct. Anal., 70, no. 2, Feb. 1987, 323-356.

[Pi2] _-, On $\mathrm{U}(\infty)$ invariant measures, preprint.

[Pi3] _ Decomposition of regular representations for $\mathrm{U}(H)_{\infty}$, Pacific J. Math., 128, no. 2 (1987), 319-332.

[Pi4] - Separable representations of automorphism groups of infinite symmetric spaces, J. Funct. Anal., 90, no. 1, (1990), 1-26.

[Pi5] _ On the Michelsson-Faddeev extension and unitary representations, Comm. Math. Phys., 123 (1989), 617-625.

[Pi6] _- Harmonic analysis on infinite dimensional symmetric spaces, to appear.

[Se] I. Segal, The structure of a class of representations of the unitary group on a Hilbert space, Proc. Amer. Math. Soc., 8 (1957), 197-203.

[Se2] G. Segal, The definition of conformal field theory, notes.

[Y] Y. Yamasaki, Lecture notes on measures on infinite dimensional spaces. 
[MR] J. Mickelsson and S. Rajeev, Current algebras in $d+1$ dimensions and determinant bundles over infinite dimensional Grassmannians, Comm. Math. Physics, 116 (1988), 365-385.

[Mi] J. Mickelsson, Current Algebras and Groups, Plenum Press, London and New York (1989).

Received April 10, 1989.

UNIVERSITY OF ARIZONA

TUCSON, AZ 85721 


\section{PACIFIC JOURNAL OF MATHEMATICS EDITORS}

\author{
V. S. VARADARAJAN \\ (Managing Editor) \\ -University of California \\ Los Angeles, CA 90024-1555-05 \\ Herbert Clemens \\ University of Utah \\ Salt Lake City, UT 84112 \\ THOMAS ENRIGHT \\ University of California, San Diego \\ La Jolla, CA 92093
}

R. FINN

Stanford University

Stanford, CA 94305

Hermann FlaschKa

University of Arizona

Tucson, AZ 85721

VAUGHAN F. R. JONES

University of California

Berkeley, CA 94720

SteVen KerCKhoff

Stanford University

Stanford, CA 94305
C. C. MOORE

University of California

Berkeley, CA 94720

Martin ScharlemanN

University of California

Santa Barbara, CA 93106

\section{HAROLD STARK}

University of California, San Diego

La Jolla, CA 92093

\section{ASSOCIATE EDITORS}

\begin{tabular}{|c|c|c|c|c|}
\hline R. ARENS & $\begin{array}{l}\text { E. F. BECKENBACH } \\
(1906-1982)\end{array}$ & NeUManN & $\begin{array}{l}\text { F. WoLF } \\
(1904-1989)\end{array}$ & K. YoshidA \\
\hline \multicolumn{5}{|c|}{ SUPPORTING INSTITUTIONS } \\
\hline \multicolumn{2}{|c|}{ UNIVERSITY OF ARIZONA } & \multicolumn{3}{|c|}{ UNIVERSITY OF OREGON } \\
\hline \multirow{2}{*}{\multicolumn{2}{|c|}{$\begin{array}{l}\text { UNIVERSITY OF BRITISH COLUMBIA } \\
\text { CALIFORNIA INSTITUTE OF TECHNOLOGY }\end{array}$}} & \multicolumn{3}{|c|}{ UNIVERSITY OF SOUTHERN CALIFORNIA } \\
\hline & & \multicolumn{3}{|c|}{ STANFORD UNIVERSITY } \\
\hline CALIFORNIA INSTITUTE OF TECHNOLOGY & UNIVERSITY OF CALIFORNIA & \multicolumn{3}{|c|}{ UNIVERSITY OF HAWAII } \\
\hline \multicolumn{2}{|c|}{ MONTANA STATE UNIVERSITY } & \multicolumn{3}{|c|}{ UNIVERSITY OF TOKYO } \\
\hline \multicolumn{2}{|c|}{ UNIVERSITY OF NEVADA, RENO } & \multicolumn{3}{|c|}{ UNIVERSITY OF UTAH } \\
\hline \multicolumn{2}{|c|}{ NEW MEXICO STATE UNIVERSITY } & \multicolumn{3}{|c|}{ WASHINGTON STATE UNIVERSITY } \\
\hline OREGON STA & UNIVERSITY & UNIVERS & OF WASHING & \\
\hline
\end{tabular}




\section{Pacific Journal of Mathematics}

Vol. 150, No. $1 \quad$ September, 1991

Jan Aarts, Charles Lemuel Hagopian and Lex Gerard Oversteegen, The orientability of matchbox manifolds $\ldots \ldots \ldots \ldots \ldots \ldots \ldots \ldots \ldots \ldots \ldots \ldots$

Roger W. Barnard, Carl Hanson Fitzgerald and Sheng Gong, The growth and $1 / 4$-theorems for starlike mappings in $\mathbf{C}^{n} \ldots \ldots \ldots \ldots \ldots \ldots \ldots$

Allan Berele, Trace rings for verbally prime algebras $\ldots \ldots \ldots \ldots \ldots \ldots \ldots 23$

Quo-Shin Chi, Curvature characterization and classification of rank-one

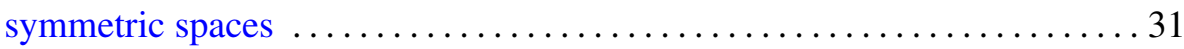

Harold Gerard Donnelly and Jeffrey Marc Lee, Domains in Riemannian

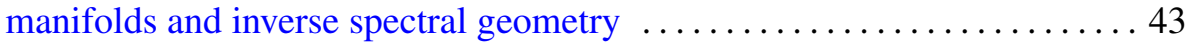

Clifford John Earle, Jr. and Patricia Lilaine Sipe, Families of Riemann

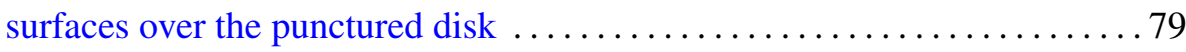

Mark P. Hughes, Dihedral group actions on homotopy complex projective

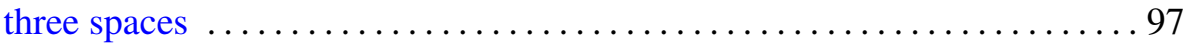

Robert F. Lax and Carl Widland, Gap sequences at a singularity ........111

Takahiko Nakazi, Bounded Hankel forms with weighted norms and lifting

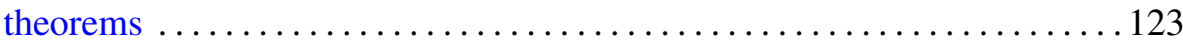

Douglas Murray Pickrell, Mackey analysis of infinite classical motion groups

Martha Rzedowski-Calderón and Gabriel Daniel Villa-Salvador,

Automorphisms of congruence function fields 167

Peter N-S Wong, Equivariant Nielsen fixed point theory for $G$-maps 